(2) Open Access Full Text Article

\title{
Younger age, female sex, and high number of awakenings and arousals predict fatigue in patients with sleep disorders: a retrospective polysomnographic observational study
}

\author{
This article was published in the following Dove Press journal: \\ Neuropsychiatric Disease and Treatment \\ 27 September 2013 \\ Number of times this article has been viewed
}

\author{
Christian Veauthier ${ }^{1,2}$ \\ 'Department of Neurology, Hanse- \\ Klinikum, Stralsund, Germany; \\ 2Department of Neurology, Centre \\ Hospitalier de Belfort-Montbéliard \\ (CHBM), Montbéliard, France
}

Correspondence: Christian Veauthier Centre Hospitalier de BelfortMontbéliard (CHBM), 2 Rue du Dr Flamand, 252II Montbéliard, France Tel +33 680756030

Fax +4932I 2828668

Email cveauthier@web.de
Background: The Fatigue Severity Scale (FSS) is widely used to assess fatigue, not only in the context of multiple sclerosis-related fatigue, but also in many other medical conditions. Some polysomnographic studies have shown high FSS values in sleep-disordered patients without multiple sclerosis. The Modified Fatigue Impact Scale (MFIS) has increasingly been used in order to assess fatigue, but polysomnographic data investigating sleep-disordered patients are thus far unavailable. Moreover, the pathophysiological link between sleep architecture and fatigue measured with the MFIS and the FSS has not been previously investigated.

Methods: This was a retrospective observational study $(n=410)$ with subgroups classified according to sleep diagnosis. The statistical analysis included nonparametric correlation between questionnaire results and polysomnographic data, age and sex, and univariate and multiple logistic regression.

Results: The multiple logistic regression showed a significant relationship between FSS/ MFIS values and younger age and female sex. Moreover, there was a significant relationship between FSS values and number of arousals and between MFIS values and number of awakenings.

Conclusion: Younger age, female sex, and high number of awakenings and arousals are predictive of fatigue in sleep-disordered patients. Further investigations are needed to find the pathophysiological explanation for these relationships.

Keywords: fatigue, sleep disorders, multiple sclerosis, polysomnography, Modified Fatigue Impact Scale, Fatigue Severity Scale, nocturnal awakening, age

\section{Introduction}

Fatigue is the most frequent symptom in multiple sclerosis (MS). ${ }^{1}$ The Multiple Sclerosis Council defines MS related fatigue as a "subjective lack of physical and/or mental energy that is perceived by the individual or caregiver to interfere with usual and desired activities,"2 and has recommended evaluation with the 21-item Modified Fatigue Impact Scale (MFIS) to measure fatigue, ${ }^{2}$ which is a smaller version of the 40-item Fatigue Impact Scale (FIS). ${ }^{3}$

Krupp et al, ${ }^{4}$ in 1989, published the unidimensional Fatigue Severity Scale (FSS), which is widely used not only in MS, but also in stroke, ${ }^{5}$ Parkinson's disease, ${ }^{5}$ lung diseases, ${ }^{6}$ myotonic dystrophy, ${ }^{7}$ neuroborreliosis, ${ }^{8} \mathrm{HIV}$ infection, ${ }^{9}$ and cancer, ${ }^{10}$ and in sleep disorders such as insomnia, ${ }^{11}$ shift working, ${ }^{12}$ restless legs syndrome (RLS), ${ }^{13,14}$ and obstructive sleep apnea. ${ }^{15}$ 
Table I Age and polysomnographic data

\begin{tabular}{|c|c|c|c|c|c|c|c|c|c|c|c|c|}
\hline & \multicolumn{3}{|c|}{ All patients } & \multicolumn{3}{|c|}{ Untreated SBRD } & \multicolumn{3}{|c|}{ Treated SRBD } & \multicolumn{3}{|c|}{ PLMD/RLS } \\
\hline & All & M & $\mathbf{F}$ & All & $M$ & $\mathbf{F}$ & All & $M$ & $\mathbf{F}$ & $\overline{\text { All }}$ & $M$ & $\mathbf{F}$ \\
\hline Number & 410 & 290 & 120 & 231 & 176 & 55 & 102 & 80 & 22 & 19 & 6 & 13 \\
\hline$\%$ & 100 & 70.7 & 29.3 & 56.3 & 42.9 & 13.4 & 24.9 & 19.5 & 5.4 & 4.7 & 1.5 & 3.2 \\
\hline \multicolumn{13}{|c|}{ Age (years) } \\
\hline Mean & 58.1 & 58.3 & 57.6 & 59.0 & 58.4 & 60.9 & 61.0 & 61.1 & 60.7 & 52.1 & 47.5 & 54.2 \\
\hline SEM & 0.7 & 0.6 & 0.6 & 0.6 & 0.7 & 1.2 & 0.6 & 0.7 & 1.2 & 0.6 & 0.1 & 1.2 \\
\hline $25 \%-75 \%$ & $51.0-68.0$ & $51.0-68.0$ & $51.0-69.0$ & $52.0-68.0$ & $52.0-66.0$ & $53.0-72.0$ & $54.0-69.0$ & $53.8-69.0$ & $54.0-69.5$ & $42.0-56.0$ & $38.5-58.0$ & $51.0-57.0$ \\
\hline \multicolumn{13}{|c|}{ AHI/hour } \\
\hline Mean & 20.0 & 21.7 & 15.7 & 32.3 & 33.3 & 29.2 & 3.4 & 2.7 & 5.7 & 3.5 & 5.0 & 1.3 \\
\hline SEM & 1.1 & 1.3 & 2.0 & I.I & 1.3 & 2.0 & I.I & 1.3 & 2.0 & I.I & 1.6 & 2.0 \\
\hline $25 \%-75 \%$ & $|.5-3| . \mid$ & $1.6-34.6$ & $||-2 \mid .8$. & $18.6-42.9$ & $19.6-42.8$ & $10.4-43.8$ & $0.2-1.6$ & $0.2-1.6$ & $0.2-2.1$ & $1.0-4.7$ & $1.0-4.7$ & $1.0-4.5$ \\
\hline \multicolumn{13}{|c|}{ SpO2\% min } \\
\hline Mean & 82.9 & 82.9 & 84.5 & 78.0 & 78.8 & 78.6 & 89.6 & 89.7 & 89.5 & 87.5 & 85.9 & 88.2 \\
\hline SEM & 0.5 & 0.6 & 0.8 & 0.5 & 0.6 & 1.0 & 0.5 & 0.6 & 1.0 & 0.5 & 0.7 & 1.0 \\
\hline $25 \%-75 \%$ & $80.0-90.0$ & $79.0-90.0$ & $84.0-91.0$ & $74.8-86.0$ & $75.0-86.0$ & $74.8-87.0$ & $88.0-92.0$ & $88.0-92.0$ & $87.8-92.0$ & $85.0-92.0$ & $78.2-92.3$ & $86.0-93.0$ \\
\hline \multicolumn{13}{|c|}{ Sleep efficacy (\%TST/TiB) } \\
\hline Mean & 72.3 & 72.2 & 72.5 & 72.2 & 72.9 & 69.7 & 71. & 70.8 & 72.8 & 73.8 & 66.0 & 77.5 \\
\hline SEM & 0.6 & 0.8 & 1.0 & 0.6 & 0.8 & I.I & 0.6 & 0.8 & I.I & 0.7 & 1.0 & 1.1 \\
\hline $25 \%-75 \%$ & $66.5-80.8$ & $66.5-80.9$ & $66.4-80.5$ & $66.8-80.9$ & $68.0-81.3$ & $60.5-77.9$ & $64.2-78.2$ & $63.3-78.3$ & $64.9-78.7$ & $70.7-85.7$ & $55.3-81.7$ & $71.6-86.0$ \\
\hline \multicolumn{13}{|c|}{ NREM-3/4 (\%/TiB) } \\
\hline Mean & 12.4 & 9.9 & 17.9 & 7.5 & 6.1 & 12.0 & 18.5 & 16.4 & 26.0 & 25.0 & 28.4 & 23.5 \\
\hline SEM & 0.6 & 0.6 & 1.2 & 0.6 & 0.6 & 1.2 & 0.6 & 0.6 & 1.2 & 0.6 & 0.8 & 1.2 \\
\hline $25 \%-75 \%$ & I.2-20.2 & $0.8-16.8$ & $7.0-25.4$ & $0.5-11.9$ & $0.3-8.8$ & $1.2-19.0$ & $9.2-25.2$ & $8.2-24.6$ & $18.3-34.2$ & | $5.6-29.7$ & $5.1-44.1$ & $16.6-30.3$ \\
\hline \multicolumn{13}{|c|}{ Number of awakenings (per night) } \\
\hline Mean & 11.8 & 10.8 & 14.4 & 1.0 & 10.6 & 12.8 & 8.7 & 8.6 & 9.2 & 24.4 & 25.5 & 23.9 \\
\hline SEM & 0.5 & 0.5 & 1.2 & 0.5 & 0.5 & 1.2 & 0.5 & 0.5 & 1.2 & 0.5 & 0.7 & 1.2 \\
\hline $25 \%-75 \%$ & $6.3-13.0$ & $6.0-11.0$ & $7.0-18.0$ & $6.0-12.0$ & $6.0-11.0$ & $6.8-14.3$ & $6.0-9.0$ & $6.0-9.0$ & $6.0-9.3$ & $|5.0-3| .0$ & $|8.5-3| .8$ & 13.5-33.5 \\
\hline \multicolumn{13}{|c|}{ Arousal index (per hour) } \\
\hline Mean & 25.1 & 26.5 & 20.6 & 47.0 & 30.4 & 25.2 & 19.8 & 20.9 & 15.7 & 19.2 & 22.1 & 17.8 \\
\hline SEM & 0.7 & 0.8 & I.I & 0.7 & 0.8 & 1.2 & 0.7 & 0.9 & 1.2 & 0.7 & 1.0 & 1.2 \\
\hline $25 \%-75 \%$ & $15.0-30.6$ & $16.1-32.3$ & $12.5-25.6$ & $28.0-59.5$ & $19.3-38.9$ & $14.3-30.0$ & $13.3-23.9$ & |4.3-24.5 & $|0.9-2| . \mid$ & |3.5-22.| & $18.8-26.4$ & $12.6-2 \mid .8$ \\
\hline \multicolumn{13}{|c|}{ Sleep latency (minutes) } \\
\hline Mean & 45.5 & 44.2 & 48.7 & 29.0 & 46.1 & 50.2 & 43.4 & 40.4 & 54.7 & 32.5 & 27.4 & 34.9 \\
\hline SEM & 1.4 & 1.6 & 3.0 & 1.4 & 1.6 & 0.3 & 1.4 & 1.6 & 3.0 & 0.1 & 2.0 & 3.0 \\
\hline $25 \%-75 \%$ & $27.0-58.0$ & $27.0-56.0$ & $26.3-62.8$ & $18.6-36.3$ & $28.0-58.0$ & $29.8-62.3$ & $28.9-52.8$ & $27.0-50.8$ & $35.0-80.3$ & $16.0-47.0$ & $13.4-36.3$ & $16.0-52.0$ \\
\hline
\end{tabular}

Abbreviations: AHI, apnea/hypopnea index; NREM-3/4, non-rapid eye movement stage 3/4; PLMD, periodic limb movement disorder; RLS, restless legs syndrome; SEM, standard error of the mean; SpO2\% min, minimal desaturation; SRBD, sleep-related breathing disorder; TiB, time in bed; TST, total sleep time; F, female; M, male.

The International Classification of Sleep Disorders second edition (ICSD-2) mentions "exhaustion" as a minimal criterion of sleep apnea, ${ }^{16}$ but in general there are no objective measurements to differentiate fatigue from exhaustion. Complaints of fatigue may be as important as those of sleepiness are to obstructive sleep apnea syndrome (OSAS) patients, ${ }^{17}$ and consequently, continuous positive airway pressure (CPAP) improves fatigue substantially in OSAS patients. ${ }^{18}$ (Interestingly, women suffering from OSAS appear to have complaints of fatigue more frequently than men with OSAS). ${ }^{17}$

Hossain et a ${ }^{19}$ investigated 283 patients by polysomnography in a sleep laboratory. Sixty-four percent of referred patients reported pathological fatigue without overlap of sleepiness (mean FSS 5.1; mean Epworth Sleepiness Scale
$[\mathrm{ESS}]^{20} 6.9$ ), 19\% were highly fatigued and sleepy (mean FSS 5.4; mean ESS 17.2), 13\% did not feel either fatigue or sleepiness (mean FSS 2.1; mean ESS 5.4), and only $4 \%$ reported pathological sleepiness without overlap of fatigue (mean FSS 1.9; mean ESS 14.5).

In summary, fatigue and sleepiness can occur together, but fatigue and sleepiness can be independent manifestations of a sleep disorder; ${ }^{21}$ there are many patients suffering from sleep disorders with fatigue but without sleepiness. In all likelihood, fatigue is under-recognized in sleep medicine. This underemphasis may contribute to the overlooking of treatable sleep disorders, with enormous consequences for misdiagnosed patients.

Veauthier et $\mathrm{al}^{22}$ performed the first cross-sectional polysomnographic trial in the context of MS-related fatigue. 
Table I (Continued)

\begin{tabular}{|c|c|c|c|c|c|c|c|c|c|c|c|c|}
\hline & \multicolumn{3}{|l|}{ Insomnia } & \multicolumn{3}{|c|}{ Parasomnia } & \multicolumn{3}{|c|}{$\begin{array}{l}\text { Hypersomnia, narcolepsy, } \\
\text { myasthenia }\end{array}$} & \multicolumn{3}{|c|}{ No sleep disorder } \\
\hline & All & $M$ & $\mathbf{F}$ & All & $M$ & $\mathbf{F}$ & All & $M$ & $\mathbf{F}$ & All & $M$ & $\mathbf{F}$ \\
\hline Number & 22 & 6 & 16 & 3 & 2 & I & 13 & 8 & 5 & 20 & 21 & 8 \\
\hline$\%$ & 5.4 & 1.5 & 3.9 & 0.7 & 0.5 & 0.2 & 3.1 & 2.0 & 1.2 & 4.9 & 2.9 & 2.0 \\
\hline \multicolumn{13}{|c|}{ Age (years) } \\
\hline Mean & 54.3 & 55.7 & 53.8 & 69 & 68.0 & 71.0 & 44.1 & 45.7 & 41.6 & 49.6 & 50.7 & 48.0 \\
\hline SEM & 0.6 & 0.8 & 1.2 & 0.9 & I.I & 0.0 & 0.6 & 0.7 & 1.7 & 0.6 & 0.9 & 1.3 \\
\hline $25 \%-75 \%$ & $49.0-63.3$ & $41.3-70.0$ & $50.0-57.5$ & $59.0-77.0$ & $59.0-77.0$ & $71.0-71.0$ & $33.0-56.0$ & $32.0-56.0$ & $28.5-55.0$ & $43.5-57.0$ & $45.8-65.0$ & $42.3-57.0$ \\
\hline \multicolumn{13}{|l|}{ AHI/hour } \\
\hline Mean & 6.2 & 13.7 & 3.0 & 15.7 & 9.5 & 28.1 & 3.4 & 5.2 & 0.9 & 2.2 & 2.6 & 1.6 \\
\hline SEM & 1.1 & $\mathrm{I} .4$ & 2.1 & 1.7 & 2.1 & 0.0 & 1.1 & 1.3 & 2.6 & 1.1 & 1.5 & 2.0 \\
\hline $25 \%-75 \%$ & $0.9-5.9$ & $2.8-21.2$ & $0.6-4.8$ & $7.5-28.1$ & $7.5-11.5$ & $28.1-28.1$ & $0.4-4.3$ & $0.6-11.9$ & $0.3-2.0$ & $0.8-2.6$ & $0.9-2.6$ & $0.8-2.5$ \\
\hline \multicolumn{13}{|c|}{ SpO2\% min } \\
\hline Mean & 89.4 & 87.7 & 90.0 & 87.3 & 89.0 & 84.0 & 90.3 & 89.0 & 92.2 & 89.1 & 88.4 & 90.1 \\
\hline SEM & 0.5 & 0.6 & 1.1 & 0.8 & I.I & 0.0 & 0.5 & 0.6 & 1.4 & 0.5 & 0.7 & 1.1 \\
\hline $25 \%-75 \%$ & $88.0-91.0$ & $83.3-91.5$ & $88.0-91.3$ & $84.0-89.0$ & $89.0-89.0$ & $84.0-84.0$ & $89.3-94.0$ & $83.0-94.0$ & $89.5-94.5$ & $88.3-91.0$ & $88.5-91.0$ & $87.5-91.8$ \\
\hline \multicolumn{13}{|c|}{ Sleep efficacy (\%TST/TiB) } \\
\hline Mean & 70.9 & 67.0 & 72.6 & 72.6 & 69.8 & 78.2 & 75.9 & 72.2 & 81.2 & 76.8 & 76.9 & 76.7 \\
\hline SEM & 0.7 & 0.8 & I.I & 1.0 & 1.3 & 0.0 & 0.7 & 0.8 & 1.4 & 0.7 & 1.0 & 1.1 \\
\hline $25 \%-75 \%$ & $59.6-82.7$ & $55.2-85.5$ & $63.7-81.5$ & $60.7-78.9$ & $60.7-78.9$ & $78.2-78.2$ & $69.2-87.4$ & $63.9-83.0$ & $72.1-91.3$ & $70.6-84.5$ & $68.5-85.1$ & $71.7-83.1$ \\
\hline \multicolumn{13}{|c|}{ NREM-3/4 (\%/TiB) } \\
\hline Mean & 13.5 & 7.8 & 16.0 & 20.3 & 25.4 & 10.3 & 14.1 & 11.3 & 18.9 & 22.5 & 17.6 & 29.8 \\
\hline SEM & 0.6 & 0.7 & 1.2 & 0.9 & I.I & 0.0 & 0.6 & 0.6 & 1.6 & 0.6 & 0.7 & 1.3 \\
\hline $25 \%-75 \%$ & $5.7-21.2$ & $4.8-10.1$ & $5.7-24.4$ & $10.3-29.7$ & $21.0-29.7$ & $10.3-10.3$ & $5.6-19.3$ & $0.3-19.3$ & $15.8-23.5$ & I5.4-23.7 & $|3.5-2| .2$ & $19.0-45.8$ \\
\hline \multicolumn{13}{|c|}{ Number of awakenings (per night) } \\
\hline Mean & 20.6 & 21.7 & 20.1 & 23.3 & 24.5 & 21.0 & 17.8 & 18.1 & 17.3 & 10.7 & 10.1 & 11.5 \\
\hline SEM & 0.5 & 0.6 & 1.2 & 0.9 & I.I & 0.0 & 0.5 & 0.5 & 0.2 & 0.5 & 0.7 & 1.3 \\
\hline $25 \%-75 \%$ & $8.3-31.0$ & $7.5-35.5$ & $8.8-31.0$ & $21.0-28.0$ & $21.0-28.0$ & $21.0-21.0$ & $7.0-26.0$ & $7.0-26.0$ & $8.8-27.5$ & $6.0-14.0$ & $5.3-10.8$ & $6.5-19.5$ \\
\hline \multicolumn{13}{|c|}{ Arousal index (per hour) } \\
\hline Mean & 17.4 & 20.3 & 16.2 & 30.5 & 28.5 & 34.7 & 19.7 & 19.1 & 21.0 & 15.0 & 15.8 & 13.7 \\
\hline SEM & 0.7 & 0.9 & 1.2 & 1.0 & 1.3 & 0.0 & 0.7 & 0.8 & 1.6 & 0.7 & 1.0 & 1.3 \\
\hline $25 \%-75 \%$ & $10.8-22.6$ & II.6-29.5 & $9.8-21.2$ & $24.6-34.7$ & $24.6-32.3$ & $34.7-34.7$ & $15.1-25.6$ & $15.3-25.6$ & $12.9-33.6$ & $10.3-17.8$ & $12.2-17.4$ & $7.5-19.3$ \\
\hline \multicolumn{13}{|c|}{ Sleep latency (minutes) } \\
\hline Mean & 44.8 & 43.8 & 45.2 & 41.5 & 42.8 & 39.0 & 32.6 & 32.3 & 33.3 & 58.7 & 58.3 & 59.3 \\
\hline SEM & 1.5 & 1.8 & 3.2 & 2.4 & 2.6 & 0.0 & 0.5 & 1.7 & 4.2 & 1.5 & 2.1 & 3.2 \\
\hline $25 \%-75 \%$ & $21.0-58.9$ & $16.8-74.5$ & $21.0-49.3$ & $26.0-59.5$ & $26.0-59.5$ & $39.0-39.0$ & $10.0-44.0$ & $10.0-44.0$ & $7.8-61.0$ & $41.8-69.8$ & $37.0-59.5$ & $47.8-75.8$ \\
\hline
\end{tabular}

They found a significant relationship between relevant sleep disorders (including sleep-related breathing disorders [SRBDs], RLS, periodic limb movement disorder [PLMD], insomnia, and others) on the one hand and fatigue measured with the MFIS and the FSS on the other hand (mean MFIS in fatigued MS patients 42.8 [standard deviation $\{\mathrm{SD}\} 18.3$ ] and 20.5 [SD 17] in non-fatigued patients $[P<0.001])$. After performing a receiver operating characteristic analysis, ${ }^{23}$ they recommended polysomnographic investigations in all fatigued MS patients, when MFIS > 34 or Pittsburgh Sleep Quality Index (PSQI) ${ }^{24}>5$, so as not to overlook treatable sleep disorders. ${ }^{23}$

Two different sleep studies have shown a significant relationship between MS-related fatigue and sleep disorders, ${ }^{22,25}$ and, in the corresponding follow-up trials, medical sleep treatment led to an improvement of MS-related fatigue. $^{26,27}$

Aside from the abovementioned studies, ${ }^{10,11,19,21}$ there are only a few polysomnographic studies investigating fatigue measured with the FSS, ${ }^{28,29}$ and there are no other polysomnographic studies investigating fatigue measured with the MFIS in the general population.

Hossain et al described FSS mean values from 4.4 (SD 1.6) to 5.1 (SD 1.0) in OSAS, RLS, PLMD, depression, narcolepsy, parasomnia, delayed sleep phase syndrome, and insomnia, ${ }^{19}$ but as there have been, to date, no pathophysiological explanations for fatigue caused by sleep disorders, the question remains: is it caused by reduced sleep efficacy, disrupted sleep, suppressed deep sleep, intensified desaturation, or an increased number of hypopnea/apnea episodes? 
Table 2 Questionnaire results

\begin{tabular}{|c|c|c|c|c|c|c|c|c|c|c|c|c|}
\hline & \multicolumn{3}{|c|}{ All patients } & \multicolumn{3}{|c|}{ Untreated SBRD } & \multicolumn{3}{|c|}{ Treated SRBD } & \multicolumn{3}{|c|}{ PLMD/RLS } \\
\hline & All & $\mathbf{M}$ & $\mathbf{F}$ & All & $\mathbf{M}$ & $\mathbf{F}$ & All & $M$ & $\mathbf{F}$ & All & $M$ & $\mathbf{F}$ \\
\hline Number & 410 & 290 & 120 & 231 & 176 & 55 & 102 & 80 & 22 & 19 & 6 & 13 \\
\hline$\%$ & 100 & 70.7 & 29.3 & 56.3 & 42.9 & 13.4 & 24.9 & 19.5 & 5.4 & 4.7 & 1.5 & 3.2 \\
\hline \multicolumn{13}{|l|}{ MFIS $^{2}$} \\
\hline Mean & 32.4 & 30.1 & 38.0 & 32.5 & 31.9 & 35.8 & 28.8 & 24.2 & 45.5 & 44.1 & 32.2 & 48.6 \\
\hline SEM & 1.0 & 1.2 & 1.7 & 1.0 & 1.3 & 1.8 & 1.0 & 1.3 & 1.8 & 1.0 & 1.6 & 1.8 \\
\hline $25 \%-75 \%$ & $15-49$ & $12.0-45.0$ & $23.8-52.0$ & $16-47.5$ & $15.0-45.3$ & $22.0-49.0$ & $10-45.5$ & $5.5-40.5$ & $31.5-56.3$ & $31.8-55.8$ & $23.0-57.0$ & $40.5-60.5$ \\
\hline \multicolumn{13}{|l|}{ FSS $^{4}$} \\
\hline Mean & 4.0 & 3.7 & 4.6 & 4.1 & 4.1 & 4.2 & 3.5 & 3.1 & 5.0 & 4.5 & 4.0 & 4.5 \\
\hline SEM & 0.1 & 0.1 & 0.2 & I.I & 0.1 & 0.2 & I.I & 0.1 & 0.2 & 0.1 & 0.1 & 1.2 \\
\hline $25 \%-75 \%$ & $2.2-5.7$ & $2.0-5.2$ & $3.1-6.2$ & $2.2-5.7$ & $2.2-5.7$ & $2.4-5.9$ & $2.0-5.0$ & $1.6-4.4$ & $3.8-6.7$ & $3.9-5.8$ & $2.8-5.2$ & $3.1-5.8$ \\
\hline \multicolumn{13}{|c|}{ ESS $^{20}$} \\
\hline Mean & 8.4 & 8.3 & 8.7 & 8.7 & 8.5 & 9.1 & 7.3 & 7.4 & 7.0 & 9.9 & 7.8 & 7.6 \\
\hline SEM & 0.3 & 0.4 & 0.5 & 0.4 & 0.5 & 0.5 & 0.7 & 0.6 & 3.4 & $\mathrm{I} .4$ & 0.4 & 0.5 \\
\hline $25 \%-75 \%$ & $5-11$ & $4.3-11.0$ & $5.0-11.0$ & $5-12$ & $5.0-11.0$ & $5.0-12.8$ & $4-10$ & $3.8-10.3$ & $3.5-9.0$ & $7-13.5$ & $1.8-11.8$ & $5.0-10.0$ \\
\hline \multicolumn{13}{|l|}{ PSQI $^{24}$} \\
\hline Mean & 8.2 & 7.6 & 10.0 & 8.2 & 7.9 & 9.8 & 6.7 & 6.3 & 8.4 & 11.4 & 10.2 & 12.3 \\
\hline SEM & 1.0 & 0.3 & 0.5 & 0.3 & 0.3 & 0.5 & 0.3 & 0.3 & 0.5 & 0.3 & 0.4 & 0.1 \\
\hline $25 \%-75 \%$ & $5-12$ & $4.0-11.0$ & $6.0-13.0$ & $5-11$ & $4.0-11.0$ & $7.0-13.0$ & $4-10$ & $3.0-8.0$ & $5.0-12.0$ & $8-15$ & $6.5-15.0$ & $9.0-15.0$ \\
\hline
\end{tabular}

Abbreviations: ESS, Epworth Sleepiness Scale; FSS, Fatigue Severity Scale; MFIS, Modified Fatigue Impact Scale; PLMD, periodic limb movement disorder; PSQI, Pittsburgh Sleep Quality Index; RLS, restless legs syndrome; SEM, standard error of the mean; SpO2\% min, minimal desaturation; SRBD, sleep-related breathing disorder; F, female; M, male.

The aims of this study were: to describe the MFIS values in patients admitted to a sleep laboratory and to investigate which polysomnographic parameters can predict fatigue measured with the MFIS and the FSS.

\section{Methods}

\section{Patients}

In this retrospective observational study, all consecutive patients who were admitted to the sleep laboratory of the Hanse-Klinikum, Stralsund, Germany between February 1, 2011 and January 31, 2012 were included.

\section{Questionnaires}

All patients admitted to the sleep laboratory completed the MFIS, FSS, ESS, and PSQI.

\section{Polysomnography}

Polysomnographic data were retrospectively analyzed. These whole-night measurements were made after an impedance test and a biosignal test over a period of 8 hours, comprising: $\mathrm{C} 3 / \mathrm{C} 4$ electroencephalograph (EEG) electrodes to the contralateral mastoid electrode; ground electrode; electrooculogram on the ipsilateral mastoid electrode; bipolar chin electromyogram (EMG) of the muscle mentalis or muscle submentalis (according to biosignal testing and anatomical conditions); nasal airflow (pressure sensor); thoracic and abdominal breathing (piezoelectric measurement); position sensor; snoring signal; pulse oximetry; pulse, electrocardiogram; and bipolar two-point EMG electrodes on both anterior tibial muscles.

The polysomnograph readings were scored according to the criteria of Rechtschaffen and Kales, ${ }^{30}$ using 30-second epochs. The following polysomnographic parameters were included in the analysis: apnea/hypopnea index (AHI) per hour of sleep, according to the American Academy of Sleep Medicine; ${ }^{31}$ minimal desaturation ( $\mathrm{SpO} \% \mathrm{~min}$ ); sleep efficacy (ratio total sleep time/time spent in bed); deep sleep (ratio non-rapid eye movement sleep stage 3 and 4 [NREM-3/4]/time spent in bed); number of awakenings; arousal index per hour of sleep; and sleep latency (time to first sleep epoch in minutes).

\section{Subgroups}

Sleep diagnoses were made according to the ICSD-2. ${ }^{16}$ Patients were divided for statistical reasons into different subgroups: (1) untreated SRBDs; (2) treated SRBDs (patients were treated with CPAP or bilevel therapy); (3) PLMD and RLS; (4) insomnia; (5) parasomnia; (6) other neurological diseases such as narcolepsy, hypersomnia, and MS (due to a low number of patients and small sample sizes, these patients 
Table 2 (Continued)

\begin{tabular}{|c|c|c|c|c|c|c|c|c|c|c|c|c|}
\hline & \multicolumn{3}{|c|}{ Insomnia } & \multicolumn{3}{|c|}{ Parasomnia } & \multicolumn{3}{|c|}{$\begin{array}{l}\text { Hypersomnia narcolepsy, } \\
\text { myasthenia }\end{array}$} & \multicolumn{3}{|c|}{ No sleep disorder } \\
\hline & All & $\mathbf{M}$ & $\mathbf{F}$ & All & $\mathbf{M}$ & $\mathbf{F}$ & All & $\mathbf{M}$ & $\mathbf{F}$ & All & $\mathbf{M}$ & $\mathbf{F}$ \\
\hline Number & 22 & 6 & 16 & 3 & 2 & I & 13 & 8 & 5 & 20 & 12 & 8 \\
\hline$\%$ & 5.4 & 1.5 & 3.9 & 0.7 & 0.5 & 0.2 & 3.1 & 2.0 & 1.2 & 4.9 & 2.9 & 2.0 \\
\hline \multicolumn{13}{|l|}{$\mathbf{M F I S}^{2}$} \\
\hline Mean & 33.5 & 38.7 & 31.5 & 7.7 & 2.0 & 19.0 & 40.6 & 42.5 & 37.6 & 26.1 & 19.8 & 34.8 \\
\hline SEM & I.I & 1.4 & 1.8 & 1.6 & 2.2 & 0.0 & I.I & 1.2 & 2.2 & I.I & 1.6 & 1.9 \\
\hline $25 \%-75 \%$ & $15-47.5$ & 13.8-56.3 & $15.0-44.0$ & $0.0-19.0$ & $0.0-4.0$ & $19.0-19.0$ & $22.3-57.5$ & $29.3-54.5$ & $12.0-62.0$ & $5-47$ & $0.0-34.0$ & $|6.3-5| .8$ \\
\hline \multicolumn{13}{|l|}{ FSS $^{4}$} \\
\hline Mean & 4.3 & 4.0 & 4.5 & 2.2 & 1.4 & 4.0 & 5.2 & 5.2 & 5.2 & 3.6 & 2.9 & 5.2 \\
\hline SEM & I.I & 0.1 & 0.2 & 0.2 & 0.2 & 0.0 & 0.1 & 0.1 & 0.2 & I.I & 0.2 & 0.2 \\
\hline $25 \%-75 \%$ & $2.9-5.8$ & $2.8-5.2$ & $3.1-5.8$ & $1.1-4.0$ & $1.1-1.6$ & $4.0-4.0$ & $3.7-6.6$ & $3.7-6.4$ & $2.8-6.7$ & $1.0-5.0$ & $1.0-4.1$ & $3.8-6.6$ \\
\hline \multicolumn{13}{|l|}{ ESS $^{20}$} \\
\hline Mean & 7.7 & 7.8 & 7.6 & 3.0 & 3.0 & 3.0 & 12.7 & 11.6 & 15.3 & 8.9 & 8.4 & 9.5 \\
\hline SEM & 0.8 & 0.4 & 0.1 & 0.7 & 0.6 & 0.0 & 1.3 & 0.4 & 0.3 & I.I & 0.5 & 0.1 \\
\hline $25 \%-75 \%$ & $4-10$ & $1.8-11.8$ & $5.0-10.0$ & $2.0-4.0$ & $2.0-4.0$ & $3.0-3.0$ & $7.8-16.0$ & $7.0-16.0$ & $14.0-16.0$ & $7.0-10.5$ & $7.0-9.5$ & $4.0-14.0$ \\
\hline \multicolumn{13}{|l|}{ PSQI $^{24}$} \\
\hline Mean & 13.2 & 13.3 & 13.1 & 4.5 & 3.0 & 6.0 & 10.6 & 12.0 & 5.0 & 7.9 & 7.1 & 8.8 \\
\hline SEM & 0.3 & 0.3 & 0.5 & 0.4 & 0.1 & 0.0 & 0.3 & 0.3 & 0.0 & 0.3 & 0.4 & 0.5 \\
\hline $25 \%-75 \%$ & $11-15$ & $|3.0-| 4.0$ & $11.0-15.0$ & $3.0-6.0$ & $3.0-3.0$ & $6.0-6.0$ & $7.0-14.5$ & $9.3-15.3$ & $5.0-5.0$ & $5.0-11.3$ & $4.3-1 \mid .5$ & $5.0-12.5$ \\
\hline
\end{tabular}

were classified into one subgroup even though these diseases are quite different); and (7) patients admitted to the sleep laboratory for tiredness or sleepiness but without pathological findings in the medical sleep investigations (exclusion of a sleep disorder).

\section{Statistical analysis}

The results were expressed as mean, SD, and range. Following an exploratory analysis of the data and after a (negative) check for normality of the underlying distributions, differences between subgroups with respect to MFIS, ESS, PSQI, and FSS were (univariately) analyzed using the Mann-Whitney $U$ test. Spearman correlation coefficients were calculated to assess the relationship between questionnaire results, and polysomnographic parameters and age, and scatter plots were performed to demonstrate these relationships. We defined Spearman's rank correlations (Spearman's rho) as follows: for values between 0.9 and 1 , the correlation is very strong; for values between 0.7 and 0.89 , the correlation is strong; values between 0.5 and 0.69 show a moderate correlation; for values between 0.3 and 0.49 , the correlation is moderate to low; and for values $<0.3$, the correlation is weak and not important.

Patients were classified into subgroups by MFIS (MFIS greater versus less than or equal to MFIS mean) and FSS
(FSS greater versus less than or equal to FSS mean) values. Afterwards, a logistic regression was performed in order to predict the outcome of these two dependent variables (greater versus less than or equal to FSS mean/MFIS mean) with age, sex, and polysomnographic parameters, including AHI, SpO2\% min, sleep efficacy, NREM-3/4, number of awakenings, arousal index, and sleep latency, as predictor variables. This analysis was performed in two steps: first, a univariate analysis with all predictor variables; subsequently, all significant variables remained in the final model of multiple logistic. Odds ratios were calculated. Statistical significance was established at $P<0.05$.

All tests should be understood as constituting exploratory data analysis, such that neither previous power calculations nor subsequent adjustments for multiple testing have been made. Analysis was performed with SPSS software (v19; IBM Corporation, Armonk, NY, USA).

\section{Results \\ Demographics, polysomnographic data, and questionnaire results}

In sum, 410 patients 20 to 86 years ( 290 men and 120 women) were investigated. Table 1 displays the demographic and polysomnographic data and Table 2 shows the questionnaire results. 
Table 3 Univariate nonparametric correlations between questionnaires, and age and PSG parameters

\begin{tabular}{|c|c|c|c|c|c|c|c|c|}
\hline & \multicolumn{2}{|l|}{ FSS $^{4}$} & \multicolumn{2}{|l|}{ MFIS $^{2}$} & \multicolumn{2}{|l|}{ ESS $^{20}$} & \multicolumn{2}{|l|}{ PSQI $^{24}$} \\
\hline & $\begin{array}{l}\text { Spearman's } \\
\text { rho }\end{array}$ & $\begin{array}{l}\text { Significance } \\
(P)\end{array}$ & $\begin{array}{l}\text { Spearman's } \\
\text { rho }\end{array}$ & $\begin{array}{l}\text { Significance } \\
(P)\end{array}$ & $\begin{array}{l}\text { Spearman's } \\
\text { rho }\end{array}$ & $\begin{array}{l}\text { Significance } \\
(P)\end{array}$ & $\begin{array}{l}\text { Spearman's } \\
\text { rho }\end{array}$ & $\begin{array}{l}\text { Significance } \\
(P) \\
\end{array}$ \\
\hline \multicolumn{9}{|l|}{ All patients } \\
\hline \multicolumn{9}{|l|}{ Scale } \\
\hline FSS & & & 0.742 & $<0.0001$ & 0.289 & $<0.0001$ & 0.488 & $<0.0001$ \\
\hline MFIS & 0.742 & $<0.0001$ & & & 0.382 & $<0.0001$ & 0.520 & $<0.0001$ \\
\hline ESS & 0.289 & $<0.0001$ & 0.382 & $<0.0001$ & & & 0.202 & 0.006 \\
\hline PSQI & 0.488 & $<0.0001$ & 0.520 & $<0.0001$ & 0.202 & 0.006 & & \\
\hline \multicolumn{9}{|l|}{ PSG } \\
\hline $\mathrm{AHI}$ & 0.098 & 0.065 & 0.077 & 0.136 & 0.047 & 0.457 & 0.132 & 0.031 \\
\hline $\mathrm{SpO} 2 \% \min$ & -0.047 & 0.374 & -0.025 & 0.634 & 0.018 & 0.783 & -0.078 & 0.207 \\
\hline Sleep efficacy & -0.005 & 0.921 & 0.010 & 0.839 & 0.078 & 0.219 & -0.065 & 0.290 \\
\hline NREM-3/4 & -0.036 & 0.503 & -0.002 & 0.970 & -0.096 & 0.131 & -0.120 & 0.051 \\
\hline Awakening & 0.098 & 0.068 & 0.158 & 0.002 & 0.176 & 0.005 & 0.278 & $<0.0001$ \\
\hline Arousal index & 0.038 & $0.48 I$ & -0.059 & 0.257 & -0.056 & 0.382 & -0.052 & 0.400 \\
\hline Sleep latency & 0.053 & 0.321 & 0.006 & 0.909 & -0.065 & 0.306 & 0.063 & 0.304 \\
\hline Age & -0.220 & $<0.0001$ & -0.152 & 0.003 & -0.116 & 0.067 & -0.120 & 0.050 \\
\hline \multicolumn{9}{|c|}{ Untreated SRBD patients } \\
\hline \multicolumn{9}{|l|}{ Scale } \\
\hline FSS & & & 0.686 & $<0.0001$ & 0.213 & 0.015 & 0.480 & $<0.0001$ \\
\hline MFIS & 0.686 & $<0.0001$ & & & 0.387 & $<0.0001$ & 0.469 & $<0.0001$ \\
\hline ESS & 0.213 & 0.015 & 0.387 & $<0.0001$ & & & 0.225 & 0.021 \\
\hline PSQI & 0.480 & $<0.0001$ & 0.469 & $<0.0001$ & 0.225 & 0.021 & & \\
\hline \multicolumn{9}{|l|}{ PSG } \\
\hline $\mathrm{AHI}$ & 0.092 & 0.198 & 0.065 & 0.343 & 0.113 & 0.185 & 0.076 & 0.352 \\
\hline $\mathrm{SpO} 2 \% \min$ & -0.035 & 0.622 & 0.005 & 0.940 & -0.034 & 0.689 & -0.006 & 0.943 \\
\hline Sleep efficacy & -0.006 & 0.929 & 0.076 & 0.268 & 0.076 & 0.374 & -0.046 & 0.576 \\
\hline NREM-3/4 & -0.020 & 0.776 & 0.049 & 0.478 & -0.127 & 0.137 & -0.142 & 0.083 \\
\hline Awakening & 0.061 & 0.392 & 0.120 & 0.080 & 0.152 & 0.075 & 0.253 & 0.002 \\
\hline Arousal index & 0.083 & 0.244 & -0.047 & 0.492 & -0.052 & 0.542 & 0.073 & 0.377 \\
\hline Sleep latency & 0.037 & 0.608 & -0.019 & 0.781 & -0.005 & 0.953 & 0.021 & 0.802 \\
\hline Age & -0.199 & 0.005 & -0.142 & 0.037 & -0.157 & 0.064 & -0.026 & 0.754 \\
\hline \multicolumn{9}{|c|}{ Treated SRBD patients } \\
\hline \multicolumn{9}{|l|}{ Scale } \\
\hline FSS & & & 0.830 & $<0.0001$ & 0.192 & 0.176 & 0.447 & $<000$. I \\
\hline MFIS & 0.830 & $<0.0001$ & & & 0.356 & 0.012 & 0.546 & $<000.1$ \\
\hline ESS & 0.192 & 0.176 & 0.356 & 0.012 & & & 0.131 & 0.403 \\
\hline PSQI & 0.447 & $<0.0001$ & 0.546 & $<0.000 I$ & 0.131 & 0.403 & & \\
\hline \multicolumn{9}{|l|}{ PSG } \\
\hline $\mathrm{AHI}$ & 0.044 & 0.677 & 0.080 & 0.449 & -0.084 & $0.54 I$ & 0.328 & 0.005 \\
\hline $\mathrm{SpO} 2 \% \min$ & -0.065 & 0.534 & -0.160 & 0.125 & 0.107 & 0.437 & -0.244 & 0.037 \\
\hline Sleep efficacy & -0.072 & 0.495 & -0.161 & 0.124 & -0.203 & 0.137 & -0.117 & 0.323 \\
\hline NREM-3/4 & 0.150 & 0.150 & 0.108 & 0.303 & -0.262 & 0.053 & -0.036 & 0.763 \\
\hline Awakening & 0.026 & 0.803 & 0.077 & 0.464 & 0.117 & 0.396 & 0.138 & 0.246 \\
\hline Arousal index & -0.182 & 0.080 & -0.260 & 0.012 & 0.058 & 0.672 & -0.282 & 0.016 \\
\hline Sleep latency & 0.102 & 0.332 & 0.151 & 0.148 & -0.085 & 0.539 & 0.151 & 0.201 \\
\hline Age & -0.171 & 0.102 & -0.032 & 0.759 & 0.145 & 0.291 & -0.157 & 0.184 \\
\hline \multicolumn{9}{|c|}{ PLMD and RLS patients } \\
\hline \multicolumn{9}{|l|}{ Scale } \\
\hline FSS & & & 0.325 & 0.219 & 0.303 & 0.271 & 0.089 & 0.783 \\
\hline MFIS & 0.325 & 0.219 & & & 0.272 & 0.291 & 0.452 & 0.163 \\
\hline ESS & 0.303 & 0.271 & 0.272 & 0.291 & & & -0.275 & 0.442 \\
\hline PSQI & 0.089 & 0.783 & 0.452 & 0.163 & -0.275 & 0.442 & & \\
\hline \multicolumn{9}{|l|}{ PSG } \\
\hline $\mathrm{AHI}$ & 0.055 & 0.835 & -0.040 & 0.875 & 0.240 & 0.353 & 0.444 & 0.148 \\
\hline $\mathrm{SpO} 2 \% \min$ & 0.025 & 0.925 & 0.463 & 0.053 & 0.346 & 0.173 & -0.407 & 0.189 \\
\hline
\end{tabular}


Table 3 (Continued)

\begin{tabular}{|c|c|c|c|c|c|c|c|c|}
\hline & \multicolumn{2}{|l|}{ FSS $^{4}$} & \multicolumn{2}{|l|}{ MFIS $^{2}$} & \multicolumn{2}{|l|}{ ESS $^{20}$} & \multicolumn{2}{|l|}{ PSQI $^{24}$} \\
\hline & $\begin{array}{l}\text { Spearman's } \\
\text { rho }\end{array}$ & $\begin{array}{l}\text { Significance } \\
(P)\end{array}$ & $\begin{array}{l}\text { Spearman's } \\
\text { rho }\end{array}$ & $\begin{array}{l}\text { Significance } \\
(P)\end{array}$ & $\begin{array}{l}\text { Spearman's } \\
\text { rho }\end{array}$ & $\begin{array}{l}\text { Significance } \\
(P)\end{array}$ & $\begin{array}{l}\text { Spearman's } \\
\text { rho }\end{array}$ & $\begin{array}{l}\text { Significance } \\
(P)\end{array}$ \\
\hline Sleep efficacy & 0.134 & 0.607 & 0.116 & 0.847 & 0.145 & 0.580 & -0.221 & 0.491 \\
\hline NREM-3/4 & 0.060 & 0.819 & 0.558 & 0.016 & 0.359 & 0.157 & 0.355 & 0.257 \\
\hline Awakening & -0.477 & 0.053 & 0.013 & 0.959 & 0.349 & 0.170 & -0.203 & 0.527 \\
\hline Arousal index & 0.138 & 0.599 & -0.159 & 0.528 & -0.302 & 0.239 & -0.323 & 0.305 \\
\hline Sleep latency & -0.020 & 0.938 & 0.046 & 0.856 & -0.623 & 0.008 & 0.327 & 0.299 \\
\hline Age & -0.167 & 0.522 & -0.092 & 0.717 & 0.036 & 0.890 & 0.677 & 0.016 \\
\hline \multicolumn{9}{|c|}{ Insomnia patients } \\
\hline \multicolumn{9}{|l|}{ Scale } \\
\hline FSS & & & $0.55 I$ & 0.022 & 0.474 & 0.063 & 0.267 & 0.523 \\
\hline MFIS & $0.55 I$ & 0.022 & & & 0.232 & 0.340 & -0.115 & 0.753 \\
\hline ESS & 0.474 & 0.063 & 0.232 & 0.340 & & & 0.453 & 0.221 \\
\hline PSQI & 0.267 & 0.523 & -0.115 & 0.753 & 0.453 & 0.221 & & \\
\hline \multicolumn{9}{|l|}{ PSG } \\
\hline $\mathrm{AHI}$ & -0.422 & 0.104 & -0.019 & 0.937 & -0.094 & 0.711 & 0.296 & 0.406 \\
\hline $\mathrm{SpO} 2 \% \min$ & 0.406 & 0.119 & 0.303 & 0.194 & 0.210 & 0.403 & -0.113 & 0.756 \\
\hline Sleep efficacy & -0.091 & 0.736 & -0.074 & 0.755 & 0.054 & 0.831 & 0.006 & 0.986 \\
\hline NREM-3/4 & 0.367 & 0.162 & -0.177 & 0.455 & 0.084 & 0.740 & 0.167 & 0.645 \\
\hline Awakening & 0.138 & 0.610 & 0.054 & 0.823 & 0.265 & 0.289 & 0.099 & 0.786 \\
\hline Arousal index & 0.055 & $0.84 I$ & 0.135 & 0.569 & 0.061 & 0.811 & $0.66 I$ & 0.038 \\
\hline Sleep latency & 0.610 & 0.012 & 0.145 & 0.543 & 0.286 & 0.250 & 0.605 & 0.064 \\
\hline Age & -0.100 & 0.702 & -0.091 & 0.694 & -0.150 & 0.539 & -0.258 & 0.445 \\
\hline \multicolumn{9}{|c|}{ Patients with MS, narcolepsy, hypersomnia } \\
\hline \multicolumn{9}{|l|}{ Scale } \\
\hline FSS & & & 0.914 & $<0.0001$ & 0.185 & 0.633 & 0.821 & 0.089 \\
\hline MFIS & 0.914 & $<0.0001$ & & & -0.056 & 0.877 & 0.900 & 0.037 \\
\hline ESS & 0.185 & 0.633 & -0.056 & 0.877 & & & -0.400 & 0.600 \\
\hline PSQI & 0.821 & 0.089 & 0.900 & 0.037 & -0.400 & 0.600 & & \\
\hline \multicolumn{9}{|l|}{ PSG } \\
\hline $\mathrm{AHI}$ & $-0.47 \mid$ & 0.143 & -0.102 & 0.753 & 0.366 & 0.333 & -0.600 & 0.400 \\
\hline $\mathrm{SpO} \% \mathrm{~min}$ & 0.490 & 0.126 & 0.467 & 0.125 & -0.527 & 0.145 & 0.800 & 0.200 \\
\hline Sleep efficacy & 0.132 & 0.698 & -0.133 & $0.68 I$ & 0.557 & 0.119 & 0.000 & 1.000 \\
\hline NREM-3/4 & -0.378 & 0.281 & -0.482 & 0.133 & -0.035 & 0.929 & -0.800 & 0.200 \\
\hline Awakening & 0.165 & 0.648 & -0.264 & 0.432 & 0.235 & 0.543 & 0.600 & 0.400 \\
\hline Arousal index & 0.049 & 0.894 & 0.209 & 0.537 & -0.261 & 0.497 & 0.800 & 0.200 \\
\hline Sleep latency & -0.049 & 0.894 & 0.055 & 0.873 & -0.322 & 0.398 & -0.400 & 0.600 \\
\hline Age & -0.058 & 0.857 & -0.019 & 0.950 & 0.809 & 0.005 & -0.300 & 0.624 \\
\hline \multicolumn{9}{|c|}{ Patients without sleep disorder } \\
\hline \multicolumn{9}{|l|}{ Scale } \\
\hline FSS & & & 0.890 & $<0.0001$ & 0.754 & 0.019 & 0.467 & 0.126 \\
\hline MFIS & 0.890 & $<0.0001$ & & & 0.538 & 0.135 & 0.583 & 0.029 \\
\hline ESS & 0.754 & 0.019 & 0.538 & 0.135 & & & 0.185 & 0.661 \\
\hline PSQI & 0.467 & 0.128 & 0.583 & 0.029 & 0.185 & 0.661 & & \\
\hline \multicolumn{9}{|l|}{ PSG } \\
\hline $\mathrm{AHI}$ & 0.188 & 0.503 & 0.168 & 0.493 & 0.350 & 0.356 & -0.137 & 0.640 \\
\hline $\mathrm{SpO} \%$ min & 0.075 & 0.790 & 0.329 & 0.169 & 0.249 & 0.518 & 0.064 & 0.828 \\
\hline Sleep efficacy & -0.043 & 0.878 & -0.044 & 0.858 & -0.067 & 0.864 & -0.368 & 0.196 \\
\hline NREM-3/4 & 0.595 & 0.019 & 0.480 & 0.037 & 0.506 & 0.164 & 0.146 & 0.618 \\
\hline Awakening & 0.267 & 0.336 & 0.307 & 0.202 & 0.140 & 0.720 & 0.196 & 0.501 \\
\hline Arousal index & 0.477 & 0.072 & 0.111 & 0.652 & -0.034 & 0.932 & 0.192 & 0.512 \\
\hline Sleep latency & 0.225 & 0.420 & -0.175 & 0.473 & 0.059 & $0.88 I$ & 0.363 & 0.202 \\
\hline Age & -0.229 & 0.412 & -0.186 & 0.447 & 0.204 & 0.598 & 0.003 & 0.991 \\
\hline arasomnia patie & : due to sma & mple size, $\mathrm{cc}$ & tions could & be calculated. & & & & \\
\hline
\end{tabular}

Notes: Definition of the correlations of Spearman's rho: very strong (0.9-1); strong (0.7-0.89); moderate (0.5-0.69); moderate to low $(0.3-0.4 .9)$; weak $(<0.3)$. All significant values $(P<0.05)$ with a Spearman's rho greater or equal to 0.30 (low/moderate/strong/very strong correlation) are highlighted with bold and italic.

Abbreviations: AHI, apnea/hypopnea-index; ESS, Epworth Sleepiness Scale; FSS, Fatigue Severity Scale; MFIS, Modified Fatigue Impact Scale; MS, multiple sclerosis; NREM3/4, non-rapid eye movement stage 3/4; PLMD, periodic limb movement disorder; PSG, polysomnographic; PSQI, Pittsburgh Sleep Quality Index; RLS, restless legs syndrome; Spearman's rho, Spearman's rank correlations; SPO2\% min, minimal desaturation; SRBD, sleep-related breathing disorder. 
A Correlation between arousal and MFIS

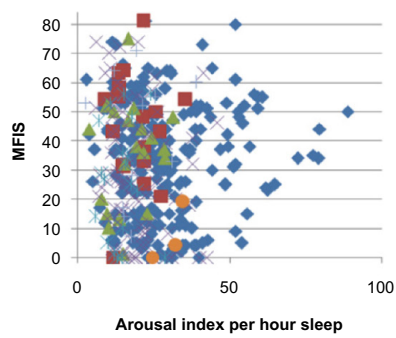

C Correlation between AHI and MFIS

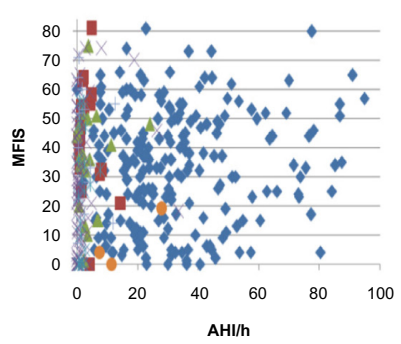

E

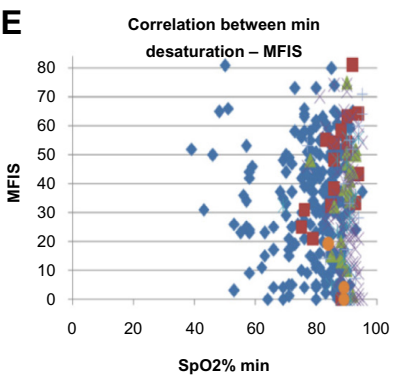

G

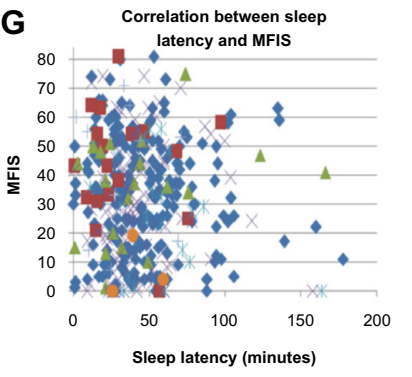

Figure I Correlation between polysomnographic parameters and MFIS ${ }^{2}$ values. Notes: Correlations between MFIS and (A) arousal; (B) awakenings; (C) AHI; (D) sleep efficacy; (E) SpO2\% min; (F) NREM-3/4; and (G) sleep latency. The scatter plots show a correlation between a high number of awakenings and high MFIS values. Note the higher amount of deep sleep in treated SRBD patients, which seems not to have an impact on MFIS or FSS values. It must also be pointed out that PLMD/RLS patients showed a high number of awakenings.

Abbreviations: AHI, apnea/hypopnea index; MFIS, Modified Fatigue Impact Scale; MS, multiple sclerosis; NREM-3/4, non-rapid eye movement sleep stage 3/4; PLMD, periodic limb movement disorder; RLS, restless legs syndrome; $\mathrm{SPO} 2 \% \mathrm{~min}$, minimal desaturation; SRBD, sleep-related breathing disorder; TiB, time in bed; TST, total sleep time; FSS, Fatigue Severity Scale.

\section{Nonparametric correlation}

Table 3 shows the nonparametric correlations.

\section{Correlations between different questionnaires}

There was a moderate to strong correlation between MFIS and FSS values in the whole cohort and in all subgroups except for the PLMD/RLS subgroup.
A Correlation between arousal and FSS

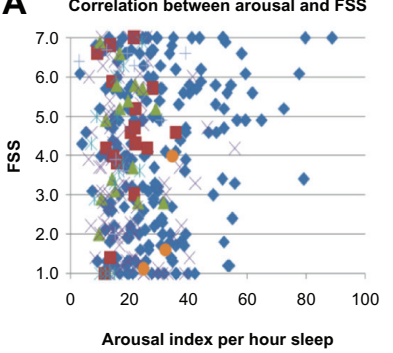

B Correlation between awakenings - FSS

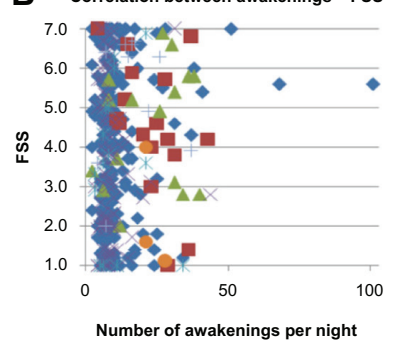

C Correlation between AHI and FSS

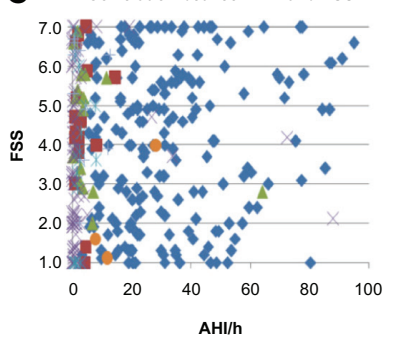

D Correlation between sleep efficacy and FsS

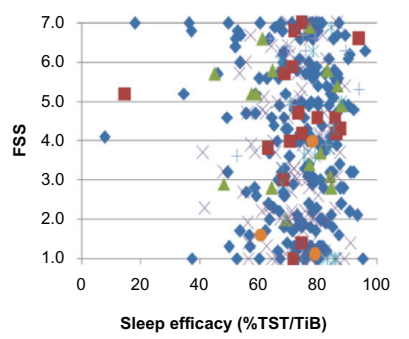

E Correlation between min desaturation - FSS

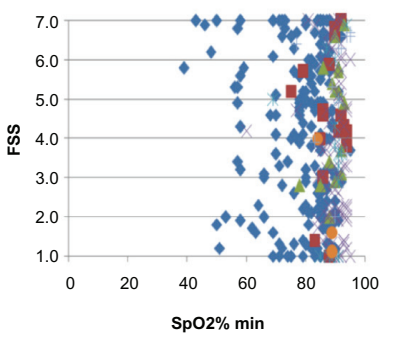

F Correlation between NREM $3 / 4$ and FSS

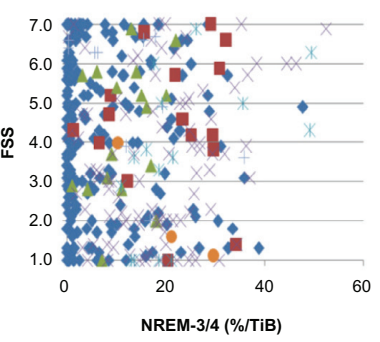

G Correlation between sleep latency and FSS

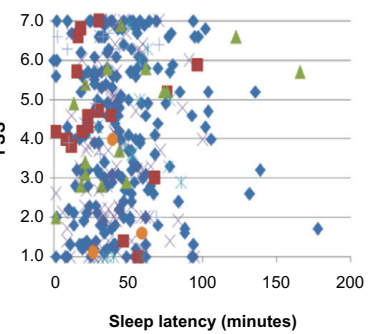

- PLMD/RLS

$\Delta$ Insomnia

$\times$ Treated SRBD

* No sleep disorder

- Parasomnia

+ Narcolepsy, hypersomnia, MS

Figure 2 Correlation between polysomnographic parameters and FSS ${ }^{4}$ values. Notes: Correlations between FSS and (A) arousal; (B) awakenings; (C) AHI; (D) sleep efficacy; (E) SpO2\% min; (F) NREM-3/4; and (G) sleep latency. The scatter plots show a correlation between a high arousal-index and high FSS values.

Abbreviations: $\mathrm{AHI}$, apnea/hypopnea index; MS, multiple sclerosis; NREM3/4, non-rapid eye movement sleep stage 3/4; PLMD, periodic limb movement disorder; RLS, restless legs syndrome; SpO2\% min, minimal desaturation; SRBD, sleep-related breathing disorder; TiB, time in bed; TST, total sleep time; FSS, Fatigue Severity Scale.

There was a moderate correlation between MFIS and PSQI in the whole cohort and in SRBD patients (treated and untreated) and in patients without sleep disorders or with narcolepsy/hypersomnia/MS (but not in PLMD/RLS patients nor insomnia patients).

There was a moderate correlation between FSS and PSQI in SRBD patients (treated and untreated). 
There was a low correlation between ESS values and MFIS in untreated SRBD patients and a moderate correlation between ESS values and FSS in patients without sleep disorders.

\section{Correlations between patient age and questionnaires}

Higher age was related to increased PSQI values in PLMD/ RLS patients and with increased ESS values in patients with narcolepsy/hypersomnia/MS (Table 3).

\section{Correlation between polysomnographic data and questionnaires}

With respect to polysomnographic data, a low correlation could be found between increased AHI and lower minimal desaturation on the one hand and increased PSQI values on the other in treated SRBD patients.

Furthermore, there was a low correlation between the number of awakenings and PSQI in all patients and untreated SRBD patients. Similarly, there was a low correlation between an increased arousal index and higher PSQI values in treated SRBD and insomnia patients.

Figures 1 and 2 show the relationships between MFIS/ FSS values and polysomnographic parameters by scatter plots. Whereas the graphical visualization did not show any correlation between MFIS/FSS values and AHI, $\mathrm{SpO} \%$ min, sleep efficacy, sleep latency, or deep sleep, the scatter plots did visualize a correlation between MFIS values and the number of awakenings and between FSS values and a high arousal index.

In the subgroup without sleep disorders, we did find a moderate nonparametric correlation between increased deep sleep (NREM-3/4) and higher MFIS and FSS values and, similarly, between deep sleep and MFIS in PLMD/RLS patients.

In PLMD/RLS patients, lower sleep latency was correlated with increased ESS values. In insomnia patients, higher FSS values were correlated with increased sleep latency.

\section{Univariate and multiple logistic regressions}

We introduced the dichotomous variable "MFIS higher than MFIS mean (34.2)" (yes or no) and the dichotomous variable "FSS higher than FSS mean (4.0)" (yes or no) in order to calculate a multiple logistic regression.

The univariate logistic regression showed no significant relationship with $\mathrm{SpO} \%$ min, sleep efficacy, sleep latency, or deep sleep. A significant relationship could be found with age, sex, and number of awakenings for MFIS as well as FSS (Table 4). Furthermore, there was a significant relationship with arousal index and AHI only for FSS.
The multiple logistic regression (Table 4) investigating the FSS, including age, sex, arousal index, number of awakenings, and AHI, demonstrated a significant relationship with age, sex, and arousal index, whereas AHI and number of awakenings were not significant. Younger age, female sex, and high arousal index were highly predictive of increased FSS scores.

In regards to MFIS, the multiple logistic regression (Table 4) investigating the MFIS, including age, sex, and number of awakenings, confirmed the significant relationship with age, sex, and number of awakenings. Younger age, female sex, and high numbers of awakenings predicted high MFIS values.

\section{Discussion}

This is the first study, to our knowledge, to investigate the relationship between sleep disorders and MFIS values, and we observed a number of important findings.

Firstly, this study clearly shows that sleep disorders can lead to high MFIS values in sleep-disordered patients who are not suffering from MS.

Secondly, our results show both high MFIS and FSS values in sleep-disordered patients without MS and, above all, a strong correlation between MFIS and FSS for all patients and all subgroups except for PLMD/RLS patients. (The fact that this correlation could not be demonstrated in PLMD/ RLS patients could be due to small sample size).

Thirdly, the correlation between PSQI and MFIS values in all patients (except for PLMD/RLS and insomnia patients) and between PSQI and FSS in SRBD patients, suggests causation of fatigue by sleep disorders (the lack of correlation in some subgroups may be caused by small sample size). This could be confirmed by multiple logistic regressions, showing that a high number of awakenings were in line with a higher risk of developing fatigue measured with the MFIS and that a high arousal index increased the risk of developing fatigue measured with the FSS. This possible causative role of awakenings and arousals in the pathophysiology of fatigue is of particular interest, and further studies should be performed to elucidate its etiology.

Guilleminault et al used cyclic alternating pattern (CAP) to investigate the relationship between sleep instability and fatigue in patients with upper airway resistance syndrome (UARS) compared with healthy controls. ${ }^{32}$ They found higher arousal indexes and sleep disturbances measured with CAP analysis in patients with UARS that correlated with sleepiness and fatigue.

Feige et al summarized the hyperarousal concept in their review about sleep microstructural differences in chronic insomnia. ${ }^{33}$ They recommended spectral analysis of the 
Table 4 Univariate and multiple logistic regressions

\begin{tabular}{|c|c|c|c|c|c|c|}
\hline & \multicolumn{3}{|c|}{ Univariate logistic regressions } & \multicolumn{3}{|c|}{ Multiple logistic regressions } \\
\hline & OR & $95 \% \mathrm{Cl}$ & $P$-value & OR & $95 \% \mathrm{Cl}$ & $P$-value \\
\hline \multicolumn{7}{|l|}{ FSS $^{4}$} \\
\hline $\mathrm{AHI} /$ hour & $1.0 I I$ & $1.001-1.020$ & 0.033 & 1.005 & $0.992-1.017$ & 0.484 \\
\hline $\mathrm{SpO} 2 \% \min$ & 0.978 & $0.957-1.000$ & 0.051 & & & \\
\hline Sleep efficacy (\% TST/TiB) & 0.994 & $0.977-1.011$ & 0.459 & & & \\
\hline NREM-3/4 (\% of TiB) & 1.007 & $0.988-1.025$ & 0.479 & & & \\
\hline Number of awakenings & 1.024 & $1.000-1.049$ & 0.047 & 1.018 & $0.993-1.043$ & 0.155 \\
\hline Arousal index/hour & 1.020 & $1.004-1.036$ & 0.017 & 1.022 & $1.00 I-I .044$ & 0.043 \\
\hline Sleep latency (minutes) & 1.003 & $0.995-1.012$ & 0.416 & & & \\
\hline Age (years) & 0.977 & $0.960-0.995$ & 0.010 & 0.974 & $0.956-0.992$ & 0.006 \\
\hline $\operatorname{Sex}(F / M)$ & 2.047 & $1.259-3.327$ & 0.004 & 2.387 & $1.4 \mid 8-4.020$ & 0.001 \\
\hline \multicolumn{7}{|l|}{ MFIS $^{2}$} \\
\hline $\mathrm{AHI} /$ hour & 1.008 & $0.998-1.017$ & 0.106 & & & \\
\hline $\mathrm{SpO} 2 \% \min$ & 1.002 & $0.982-1.023$ & 0.817 & & & \\
\hline Sleep efficacy (\% TST/TiB) & 1.004 & $0.989-1.020$ & 0.598 & & & \\
\hline NREM-3/4 (\% of TiB) & 1.006 & $0.988-1.023$ & 0.517 & & & \\
\hline Number of awakenings & 1.052 & $1.023-1.08 I$ & $<0.0001$ & 1.043 & $1.015-1.073$ & 0.002 \\
\hline Arousal index/hour & 1.009 & $0.994-1.024$ & 0.255 & & & \\
\hline Sleep latency (minutes) & 0.998 & $0.991-1.005$ & 0.606 & & & \\
\hline Age (years) & 0.981 & $0.964-0.964$ & 0.026 & $0.98 I$ & $0.964-0.999$ & 0.042 \\
\hline $\operatorname{Sex}(F / M)$ & 2.377 & $|.483-3.8 I|$ & $<0.0001$ & 2.108 & $I .292-3.44 \mid$ & 0.003 \\
\hline
\end{tabular}

Notes: The independent variables in the first regression include all polysomnographic parameters and age and sex. All variables that were significant in the first univariate analysis (age, sex, the number of awakenings, and for FSS values, additionally, arousal index and AHI) remained in the final model of multiple logistic regressions. The independent variables age and sex predict high FSS and MFIS values after multiple logistic regressions. In addition, a high number of awakenings predict high MFIS values, and a high arousal index predicts high FSS values. ORs were calculated, which reflect the probability to develop fatigue measured with MFIS and FSS. Bold italic font indicates significant odds ratios.

Abbreviations: AHI, apnea/hypopnea index; Cl, confidence interval; FSS, Fatigue Severity Scale; MFIS, Modified Fatigue Impact Scale; NREM-3/4, non-rapid eye movement sleep stage 3/4; OR, odds ratio; $\mathrm{SPO} 2 \%$ min, minimal desaturation; TiB, time in bed; TST, total sleep time; F, female; M, male.

sleep EEG, microarousal, and CAP analysis as a research instrument of sleep in insomnia. It would be promising to investigate fatigue as well with these methods in patients without known sleep disorders, in order to find out what drives fatigue.

On the other hand, little is known about the impact of arousal on fatigue in sleep disorders. Yue et $\mathrm{al}^{34}$ found a significant relationship between emotional fatigue measured with the Multidimensional Fatigue Symptom Inventory short form $^{35}$ and spontaneous movement arousals in untreated OSAS patients. It is worth noting that previous studies have not shown a significant association between the severity of OSAS (AHI, SpO2\% min) and fatigue scores. ${ }^{36,37}$ In our study, there was also no significant relationship between fatigue and $\mathrm{AHI}$ or $\mathrm{SpO} 2 \%$ min after multiple logistic regressions.

Previous polysomnographic investigations of consecutive MS patients showed a significant association of sleep disorders with fatigue (FSS/MFIS), ${ }^{22}$ but not with sleepiness (ESS). Supporting this finding, the works by Hossain et al ${ }^{19}$ and Aguillard et $\mathrm{al}^{21}$ showed that, in sleep-disordered patients without MS, fatigue and sleepiness can occur independently as well as together.
In the present study, there was not a significant relationship between MFIS values and ESS values (only untreated SRBD patients showed a low correlation between ESS values and MFIS). The fact that we found a strong correlation between ESS values and FSS values in patients without sleep disorders remains without explanation. We have to note that these patients were not healthy controls, but rather fatigued and sleepy patients without pathological findings either in the primary health care setting or in the sleep laboratory.

Overall, as we performed multiple logistic regressions only for MFIS and FSS values, we cannot interpret the correlations between ESS and PSQI values and age or sex or polysomnographic parameters. Our study was focused on the relationship between sleep disorders and FSS and MFIS.

In our study, women had a higher risk of suffering from fatigue than men. Our results are in line with a previous study, ${ }^{17}$ suggesting that, in SRBD patients, fatigue may be particularly correlated with female sex: in our study, female sex was predictive of high FSS and MFIS values. Currently, we have no explanation for this relationship and further studies are needed to investigate the pathophysiological mechanisms. Similarly, we have no explanation for the fact 
that younger age is predictive of fatigue and further studies are needed to understand this relationship as well.

The present study has some methodological limitations: due to the retrospective design and owing to the lack of exclusion criteria, we cannot exclude that some patients have other reasons for fatigue as a possible confounder. On the other hand, this study reflects the daily clinical praxis with consecutive patients over 1 year.

\section{Conclusion}

Younger age, female sex, and high number of awakenings and arousals are predictive of fatigue in sleep-disordered patients. Further investigations are needed to find the pathophysiological link for this relationship. This study proves that high MFIS and FSS values can occur in sleep-disordered patients.

\section{Acknowledgments}

The author thanks Dr Gosia Sullivan for reviewing the manuscript and Mrs Gerda Siebert for statistical advice.

\section{Disclosure}

The author reports no conflicts of interest in this work.

\section{References}

1. Krupp LB, Alvarez LA, LaRocca NG, Scheinberg LC. Fatigue in multiple sclerosis. Arch Neurol. 1988;45:435-437.

2. Paralyzed Veterans of America. Fatigue and Multiple Sclerosis: Evidence-Based Management Strategies for Fatigue in Multiple Sclerosis. Washington, DC: Multiple Sclerosis Council for Clinical Practice Guidelines; 1998

3. Fisk JD, Ritvo PG, Ross L, Haase DA, Marrie TJ, Schlech WF. Measuring the functional impact of fatigue: initial validation of the fatigue impact scale. Clin Infect Dis. 1994;18 Suppl 1:79-83.

4. Krupp LB, LaRocca NG, Muir-Nash J, Steinberg AD. The fatigue severity scale. Application to patients with multiple sclerosis and systemic lupus erythematosus. Arch Neurol. 1989;46(10):1121-1123.

5. Elbers RG, Rietberg MB, van Wegen E, et al. Self-report fatigue questionnaires in multiple sclerosis, Parkinson's disease and stroke: a systematic review of measurement properties. Qual Life Res. 2012;21(6):925-944.

6. Peric S, Stojanovic VR, Basta I, et al. Influence of multisystemic affection on health-related quality of life in patients with myotonic dystrophy type 1. Clin Neurol Neurosurg. 2013;115(3):270-275.

7. Ozalp O, Inal-Ince D, Calik E, et al. Extrapulmonary features of bronchiectasis: muscle function, exercise capacity, fatigue, and health status. Multidiscip Respir Med. 2012;11;7(1):3.

8. Eikeland R, Mygland A, Herlofson K, Ljøstad U. Risk factors for a non-favorable outcome after treated European neuroborreliosis. Acta Neurol Scand. 2013;127(3):154-160.

9. Lerdal A, Kottorp A, Gay C, Aouizerat BE, Portillo CJ, Lee KA. A 7-item version of the fatigue severity scale has better psychometric properties among HIV-infected adults: an application of a Rasch model. Qual Life Res. 2011;20(9):1447-1456.

10. Yun YH, Lee KS, Kim YW, et al. Web-based tailored education program for disease-free cancer survivors with cancer-related fatigue a randomized controlled trial. J Clin Oncol. 2012;30(12):1296-1303.

11. Guilleminault C, Davis K, Huynh NT. Prospective randomized study of patients with insomnia and mild sleep disordered breathing. Sleep. 2008;31(11):1527-1533.
12. Hossain JL, Reinish LW, Kayumov L, Bhuiya P, Shapiro CM. Underlying sleep pathology may cause chronic high fatigue in shift-workers. $J$ Sleep Res. 2003;12(3):223-230.

13. Cuellar NG, Strumpf NE, Ratcliffe SJ. Symptoms of restless legs syndrome in older adults: outcomes on sleep quality, sleepiness, fatigue, depression, and quality of life. JAm Geriatr Soc. 2007;55(9):1387-1392.

14. Cavalcante AG, de Bruin PF, de Bruin VM, et al. Restless legs syndrome, sleep impairment, and fatigue in chronic obstructive pulmonary disease. Sleep Med. 2012;13(7):842-847.

15. Su CS, Liu KT, Panjapornpon K, Andrews N, Foldvary-Schaefer N. Functional outcomes in patients with REM-related obstructive sleep apnea treated with positive airway pressure therapy. J Clin Sleep Med. 2012;8(3):243-247.

16. International Classification of Sleep Disorders. 2nd ed. Westchester, IL: American Academy of Sleep Medicine (AASM); 2005.

17. Chervin RD. Sleepiness, fatigue, tiredness, and lack of energy in obstructive sleep apnea. Chest. 2000;118(2):372-379.

18. Chotinaiwattarakul W, O'Brien LM, Fan L, Chervin RD. Fatigue, tiredness, and lack of energy improve with treatment for OSA. J Clin Sleep Med. 2009;5(3):222-227.

19. Hossain JL, Ahmad P, Reinish LW, Kayumov L, Hossain NK, Shapiro CM. Subjective fatigue and subjective sleepiness: two independent consequences of sleep disorders? J Sleep Res. 2005;14(3):245-253.

20. Johns MW. Reliability and factor analysis of the Epworth Sleepiness Scale. Sleep. 1992;15:376-381.

21. Aguillard RN, Riedel BW, Lichstein KL, Grieve FG, Johnson CT, Noe SL. Daytime functioning in obstructive sleep apnea patients: exercise tolerance, subjective fatigue, and sleepiness. Appl Psychophysiol Biofeedback. 1998;23(4):207-217.

22. Veauthier C, Radbruch H, Gaede G, et al. Fatigue in multiple sclerosis is closely related to sleep disorders: a polysomnographic cross-sectional study. Mult Scler. 2011;17:613-622.

23. Veauthier C, Paul F. Fatigue in multiple sclerosis: which patient should be referred to a sleep specialist? Mult Scler. 2012;18(2):248-249.

24. Buysse DJ, Reynolds CF III, Monk TH, Berman SR, Kupfer DJ. The Pittsburgh Sleep Quality Index: a new instrument for psychiatric practice and research. Psychiatry Res. 1989;28:193-213.

25. Kaminska M, Kimoff RJ, Benedetti A, et al. Obstructive sleep apnea is associated with fatigue in multiple sclerosis. Mult Scler. 2012;18(8): 1159-1169.

26. Côté I, Trojan D, Kaminska M, et al. Impact of sleep disorder treatment on fatigue in multiple sclerosis. Mult Scler. 2013;19(4):480-489.

27. Veauthier C, Gaede G, Radbruch H, Gottschalk S, Wernecke KD, Paul F. Treatment of sleep disorders may improve fatigue in multiple sclerosis. Clin Neurol Neurosurg. 2013. Epub 2013 June 10.

28. Mermigkis C, Bouloukaki I, Antoniou KM, et al. CPAP therapy in patients with idiopathic pulmonary fibrosis and obstructive sleep apnea: does it offer a better quality of life and sleep? Sleep Breath. Epub 2013 Feb 6.

29. Chaumet G, Quera-Salva MA, Macleod A, et al. Is there a link between alertness and fatigue in patients with traumatic brain injury? Neurology. 2008;71(20):1609-1613.

30. Rechtschaffen A, Kales A, editors. A Manual of Standardized Terminology Techniques and Scoring System for Sleep Stages of Human Subjects. Los Angeles, CA: US Department of Health, Education, and Welfare, Public Health Services-National Institutes of Health, National Institute of Neurological Diseases and Blindness, Neurological Information Network; 1968.

31. [No authors listed]. Sleep-related breathing disorders in adults: recommendations for syndrome definition and measurement techniques in clinical research. The Report of an American Academy of Sleep Medicine Task Force. Sleep. 1999;22:667-689.

32. Guilleminault C, Lopes MC, Hagen CC, da Rosa A. The cyclic alternating pattern demonstrates increased sleep instability and correlates with fatigue and sleepiness in adults with upper airway resistance syndrome. Sleep. 2007;30(5):641-647.

33. Feige B, Baglioni C, Spiegelhalder K, Hirscher V, Nissen C, Riemann D. The microstructure of sleep in primary insomnia: an overview and extension. Int J Psychophysiol. Epub 2013 April 11. 
34. Yue HJ, Bardwell W, Ancoli-Israel S, Loredo JS, Dimsdale JE. Arousal frequency is associated with increased fatigue in obstructive sleep apnea. Sleep Breath. 2009;13(4):331-339.

35. Stein KD, Martin SC, Hann DM, Jacobsen PB. A multidimensional measure of fatigue for use with cancer patients. Cancer Pract. 1998;6(3): $143-152$.
36. Lichstein KL, Means MK, Noe SL, Aguillard RN. Fatigue and sleep disorders. Behav Res Ther. 1997;35:733-740.

37. Bardwell WA, Moore P, Ancoli-Israel S, Dimsdale JE. Fatigue in obstructive sleep apnea: driven by depressive symptoms instead of apnea severity? Am J Psychiatry. 2003;160:350-355.

\section{Publish your work in this journal}

Neuropsychiatric Disease and Treatment is an international, peerreviewed journal of clinical therapeutics and pharmacology focusing on concise rapid reporting of clinical or pre-clinical studies on a range of neuropsychiatric and neurological disorders. This journal is indexed on PubMed Central, the 'PsycINFO' database and CAS.

The manuscript management system is completely online and includes a very quick and fair peer-review system, which is all easy to use. Visit http://www.dovepress.com/testimonials.php to read real quotes from published authors.

\footnotetext{
Submit your manuscript here: http://www.dovepress.com/neuropsychiatric-disease-and-treatment-journal
} 